Ann. Zootech., I968, $17(4), 393-407$.

\title{
ÉTUDE DU DÉTERMINISME HÉRÉDITAIRE DE L'HYPERTROPHIE MUSCULAIRE DU PORC DE PIETRAIN
}

\author{
L. OILIIVILR
}

avec la collaboration technique de P. Daxion, N. Gavpin et Helen Welsh

Station centrale de Génétique animale, Contre national de Recherches zootechniques 78 -Jouy-en-Josas

Institut national de la Recherche agronomique

\section{SOMMAIRE}

A partir d'un verrat de race de Piétrain et de onze femelles de race large White, une première génération $\left(F_{1}\right)$ de croisement a été obtenue, puis une $F_{2}\left(F_{1} \times F_{1}\right)$ et les croisements en retour $(C R)$ aux deux races parentales, afin de mettre à l'épreuve l'hypothèse d'un déterminisme monogénique de l'hypertrophie musculaire du porc de Piétrain.

Les animaux $\mathrm{F}_{1}, \mathrm{~F}_{2}$ et $\mathrm{CR}$ ont été soumis à un pointage de conformation vers l'âge de deux mois, c'est-à-dire au sevrage (406 porcs), à des mesures au cours de l'engraissement entre 25 et $100 \mathrm{~kg}$ (220 porcs), et à des mesures sur la carcasse au poids d'abattage de $100 \mathrm{~kg}$ ( 142 porcs).

Les distributions des notes de pointage suggèrent l'existence de trois catégories d'individus en deuxième génération ( $F_{2}$ et $\mathrm{CR}$ ). Par contre, aucune des mesures objectives considérée isolément ne permet de mettre en évidence des catégories distinctes. Cependant, une fonction linéaire de six mesures de carcasse - fonction discriminatoire entre les deux races parentales — présente dans s's distributions en $\mathrm{F}_{2}$ et $(\mathrm{K}$ des irrégularités analogués à celles observées pour les notes de pointage. De plus, il existe une régression significative de la fonction discriminatoire définie ci-dessus en la note de pointage attribuée au sevrage. L'ensemble de ces résultats suggère que les gènes responsables de l'hypertrophie musculaire dans la race de Piétrain sont en nombre limité, et que ces gènes s'expriment dès le moment du sevrage.

\section{INTRODUCTION}

Dès les premiers développements de la Génétique, la question du nombre de loci responsables de la variation des caractères quantitatifs a été abordée. CASTLE (I92I), proposa une méthode pour estimer ce nombre. Vers la même époque, une école de zootechniciens allemands et scandinaves avançait l'hypothèse d'un nombre restreint 
de gènes intervenant dans le déterminisme de la production laitière et du taux buty. reux (voir WRIEDT, I930). Puis, les développements de la génétique quantitative firent passer ces conceptions au second plan. Cependant, des travaux récents, sur Drosophile, ont montré que, dans certains cas, le nombre de gènes influant sur un caractère quantitatif peut être très faible (THODAY, I96r). Dans le même ordre d'idées, l'influence de certains loci bien identifiés sur des caractères de production a été étudiée et est très souvent apparue appréciable, comme dans le cas de la volaille (MÉRAT, Ig66).

On peut également chercher à connaître le nombre de gènes responsables de l'apparition de certains "types " d'animaux qu'il est difficile de caractériser par une seule mesure, tels que le "culard " dans l'espèce bovine ou le porc de Piétrain. Dans l'un et l'autre cas, l'hypothèse d'une mutation a été invoquée (pour les bovins voir LAUViRGNe et coll., ig63 et pour le pore de Piétrain voir Wili,Ems, rg60).

Dans une espèce à reproduction rapide comme le Porc, une telle hypothèse peut être mise à l'épreuve dans un délai relativement court, puisqu'il suffit théoriquement d'observer deux générations de croisement. Une expérience visant à mettre à l'épreuve l'hypothèse d'un déterminisme monogénique de l'hypertrophie musculaire du porc de Piétrain a donc été entreprise en I962 et les premiers résultats en ont été publiés (Oldivier et Lauvergne, 1967). L'expérience a été terminée en 1966 et nous présentons ici l'ensemble des résultats obtenus.

\section{PLAN E'T DÉROULEMENT DE L'EXPÉRIENCE}

L'expérience s'est déroulée au domaine de la IIaizerie à Vaux-sur-ilure (Calvados). A l'automne 1962, un verrat P'iétrain, importé de Belgique, a été accouplé à i truies de race Large While, prises dans le troupeau de la Station de Recherches sur l'Elevage des Porcs. Le plan de l'expérience prévoyait d'obtenir, à partir de ces animaux une première génération $\left(F_{1}\right)$ puis une deuxième se répartissant en trois catégories: $\mathrm{F}_{2}\left(\mathrm{~F}_{1} \times \mathrm{F}_{1}\right)$, croisement en retour au Piitrain $\left(\mathrm{F}_{1} \times\right.$ Piétrain $)$ et croisement en retour au Large White $\left(\mathbf{F}_{1} \times\right.$ Large White $)$. Tous les individus de la deuxième génération devaient avoir le même taux de consanguinité, étant le produit soit d'un croisement frère $\times$ socur $\left(\mathrm{F}_{1} \times \mathrm{F}_{1}\right)$, soit d'un croisement père $\times$ fille $\left(\mathrm{F}_{1} \times\right.$ Piétrain), soit d'un croisement mère $\times$ fils $\left(\mathrm{F}_{1} \times\right.$ Large White). Dans ce but, dans les onze portées $\mathrm{F}_{1}$ obtenues, un mâle et deux femelles ont été choisis au hasard. En fait, l'une de ces deux femelles a été accouplée à son frère de portée comme prévu mais l'autre, qui devait être accouplée à son père a dû l'être à un autre verrat Pistrain fils du précédent, mort en cours d'expérience. Enfin, chacune des truies Large White parentales a été accouplée à son fils. L'ensemble des accouplements a été répété à six mois d'intervalle, si bien que nous avons obtenu deux séries de portées $F_{1}$ (printemps et automne 1963) et trois séries de portées de deuxième génération (printemps et automne 1964, printemps 1965). tenant.

Les porcs obtenus ont été soumis à trois ensembles d’observations que nous allons décrire main-

I. Au sevrage, effectué à l'âge de deux mois, tous les porcelets ont été examinés et une note de conformation (de o à 8) a été attribuée à chacun d'eux selon le principe décrit par OLLIVIER et LAU. VERGNE (I967).

2. Un échantillon pris au hasard dans les animaux sevrés a été soumis à l'engraissement, entre les poids de 25 et de $100 \mathrm{~kg}$, dans des cases en plein air avec abris en ballots de paille. Les porcs étaient groupés à raison de I 2 par case mais alimentés individuellement en deux repas par jour (régime semi-ad libitum). La consommation journalière de chaque animal était notée et les animaux étaient pesés tous les 14 jours. En fin d'engraissement, les porcs étaient soumis à des mesures d'épaisseur de lard par la technique de IIAZEL et KLINe (1952), chaque pore étant mesuré à six endroits différents du dos (voir OLIIVIER, I964). 
Les caractères suivants ont été pris en considération :

- poids à 154 jours, obtenu par intrapolation,

- gain moyen quotidien entre les poids de 25 et de $100 \mathrm{~kg}$,

- indice de consommation entre les poids de 25 et $100 \mathrm{~kg}$,

- âge à l'abattage,

- moyenne des six mesures d'épaisseur de lard effectuées avant l'abattage.

3. Parmi les animaux engraissés, seuls ont été conservés ceux issus de la première série de misesbas de première et de deuxième génération, parmi lesquels des reproducteurs ont été gardés. Les autres ont été abattus vers le poids de $100 \mathrm{~kg}$ vif et découpés selon la méthode parisienne normalisée dans les abattoirs du C. N. R. Z. à Jouy-en-Josas.

les caractères suivants ont été analysés :

-...- poids vif à l'abattage,

- poids de la tête,

- poids de la moitié droite de la carcasse, avant découpe, panne,

- poids des différents morceaux : jambon, longe, poitrine, jambonneau, pieds, bardière et

- longueur de la carcasse suspendue, de l'atlas au bord antérieur de la symphyse pubienne,

- épaisseur de lard mesurée à la demière vertèbre lombaire (rein), à la dernière vertèbre dorsale (dos) et à la dernière vertèbre cervicale (cou).

Le tableau I donne le schéma d'ensemble de l'expérience ainsi que les effectifs soumis à chaque ensemble d'observations.

TABIEAU I

Schéma de lexpérience el effectifs contrôle's

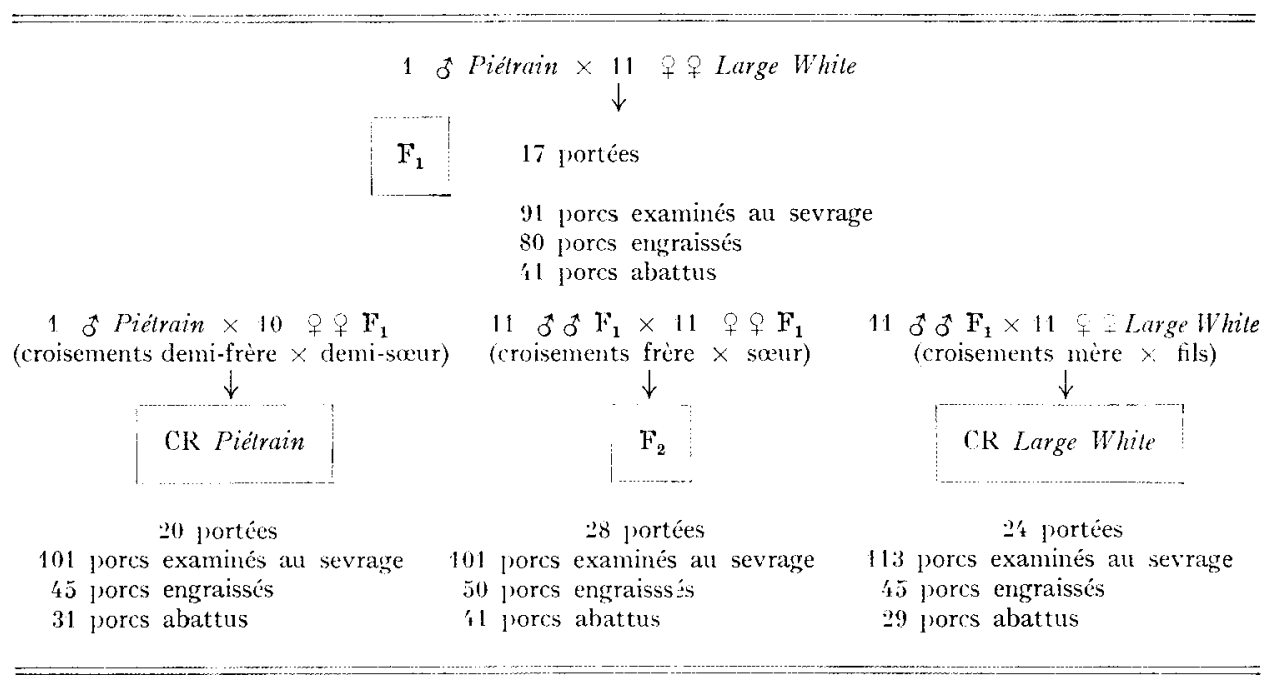

\section{MÉTHODES D'ANALYSE E'T RÉSULTATS}

\section{La conformation au sevrage}

Les moyennes des notes de pointage par type de croisement sont données dans le tableau 2. Les moyennes par sexe sont très voisines, ce qui autorise un regroupement de l'ensemble des notes en une seule distribution par type de croisement. Les 
distributions sont présentées dans la figure $\mathrm{I}$. Ces résultats, qui portent sur 406 porcs, confirment les résultats partiels obtenus sur $2 \mathrm{I} 2$ porcs et déjà rapportés précédemment (OLLIVIER et LAUVERGNE, I967).

\section{TABIEAU 2}

Moyennes des notes de pointage

\begin{tabular}{|c|c|c|c|c|}
\hline \multirow{2}{*}{ Type de croisement } & \multicolumn{3}{|c|}{ Effectif } & \multirow{2}{*}{ Moyenne } \\
\hline & Máles & Femelles & Total & \\
\hline & 49 & 51 & 91 & 5,35 \\
\hline $\mathrm{F}_{2} \ldots \ldots$ & 47 & $5^{\prime} \mathrm{t}$ & 101 & 5,45 \\
\hline $\mathrm{CK}$ Piélrain & 41 & 60 & 101 & $6,6^{\prime} \mathrm{t}$ \\
\hline CR Large White ... & 58 & 55 & 113 & 3,40 \\
\hline \multirow{3}{*}{ Total } & 186 & \multirow{3}{*}{220} & & 5,12 \\
\hline & & & & 5,19 \\
\hline & & & 406 & 5,16 \\
\hline
\end{tabular}
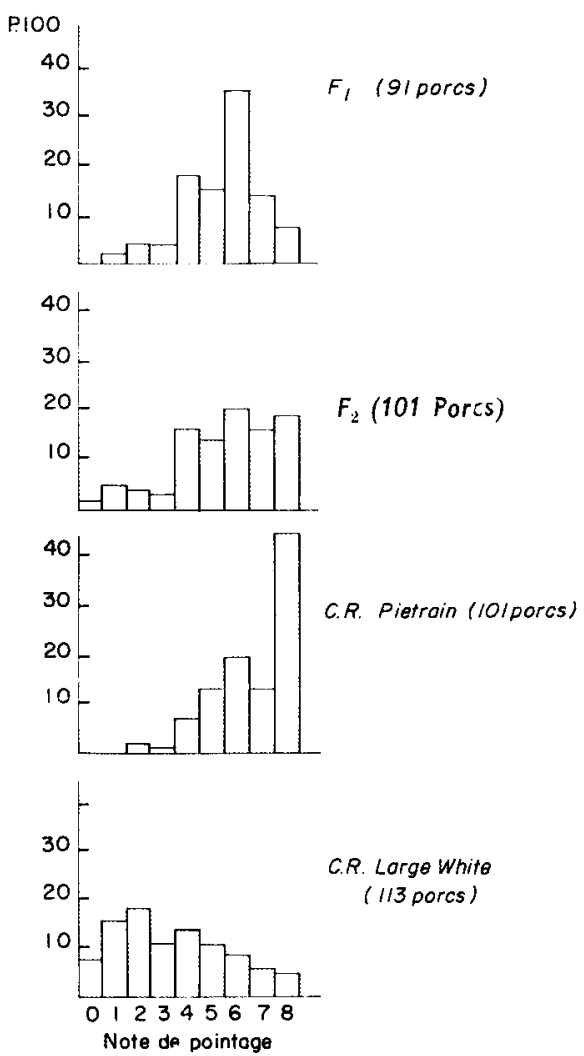

C.R. Large White ( 113 porcs)

FIG. I. - Distributions des notes de poinlage 


\section{Les mesures effectuées au cours de l'engraissement}

Quatre-vingt porcs ont été engraissés en première génération (dont un a dû être éliminé avant la fin du contrôle), répartis en deux séries, et I4o porcs en deuxième génération, répartis en trois séries. La première série dans chaque génération comprenait des mâles non castrés et des femelles, alors que dans les séries suivantes, les mâles étaient castrés. Par suite des conditions de l'élevage en plein air, des différences importantes se manifestent entre les diverses périodes d'engraissement (v. tabl. 3). Nous nous sommes, en conséquence, limités à des comparaisons intrapériode d'engraissement entre chacun des croisements en retour et la $F_{2}$. Pour les mesures d'épaisseur de lard, les comparaisons ont été réalisées intra-période et intrasexe. Les variances intra-période (et sexe pour les mesures de gras dorsal) par type de croisement sont reportées au tableau 4. Ians l'ensemble, les variances les plus faibles sont observées en $F_{1}$ et les plus élevées en $F_{2}$. Cependant, les tests d'homogénéité des variances en deuxième génération ne sont pas significatifs à l'exception de l'âge à l'abattage et de l'épaisseur du gras dorsal. On a donc supposé des variances homogènes en deuxième génération pour le poids à $\mathrm{I} 54$ jours, le gain moyen et l'indice de consommation. Pour l'âge à l'abattage et l'épaisseur du gras dorsal, on a supposé que la variance de la $F_{2}$ est le double de celle des croisements en retour. Les différences obtenues dans chaque période (ou groupe période sexe) ont été pondérées dans le premier cas par le coefficient $\frac{n_{1} n_{2}}{n_{1}+n_{2}}$, dansle second cas par $\frac{n_{1} n_{2}}{2 n_{1}+n_{2}}, n_{1}$ étant l'effectif dans le croisement en retour considéré et $n_{2}$ l'effectif en $\mathrm{F}_{2}$.

Les différences moyennes pondérées apparaissent au tableau 3. Elles sont significatives pour toutes les caractéristiques d'engraissement sauf pour l'épaisseur du gras dorsal. C'est le croisement en retour au Large White qui présente la meilleure croissance et le meilleur indice de consommation de 25 à Ioo $\mathrm{kg}$, suivi par le croisement en retour au Piétrain. Les individus $\mathrm{F}_{2}$ ont la plus mauvaise croissance et présentent, en contrepartie, moins de gras dorsal, mais sans que cette dernière différence soit significative.

\section{Mesures effectuées sur la carcasse}

Quarante et un porcs ont été abattus en $\mathrm{F}_{\mathbf{1}}$ (en une seule série) et IoI porcs en deuxième génération (en deux séries). Toutes les mesures effectuées ont d'abord été corrigées en fonction du poids vif d'abattage, à partir de la régression intra-sexegénération. Ensuite, 1'homogénéité des trois variances intra-sexe-période en deuxième génération a été vérifiée. Une seule des I3 variables (poids des pieds) manifestait une hétérogénéité significative des variances (tabl. 5). Il n'a pas été jugé utile d'en tenir compte dans la suite de l'analyse. Sur les données de la deuxième génération, les effets " sexe " " période " et " type de croisement " ont été estimés par la méthode des moindres carrés, 1'hypothèse d'additivité de ces trois effets étant compatible avec les données. Les données ont ensuite été corrigées pour le sexe et la période et ramenées sur la base de femelles engraissées pendant 1'hiver. Le tableau 6 donne les moyennes pour chaque génération, les estimations des différences entre les trois types de croisement en deuxième génération ainsi que la signification des tests $F$ relatifs à ces différences. Le type de croisement exerce un effet significatif sur le poids 
I. OLLIVIER

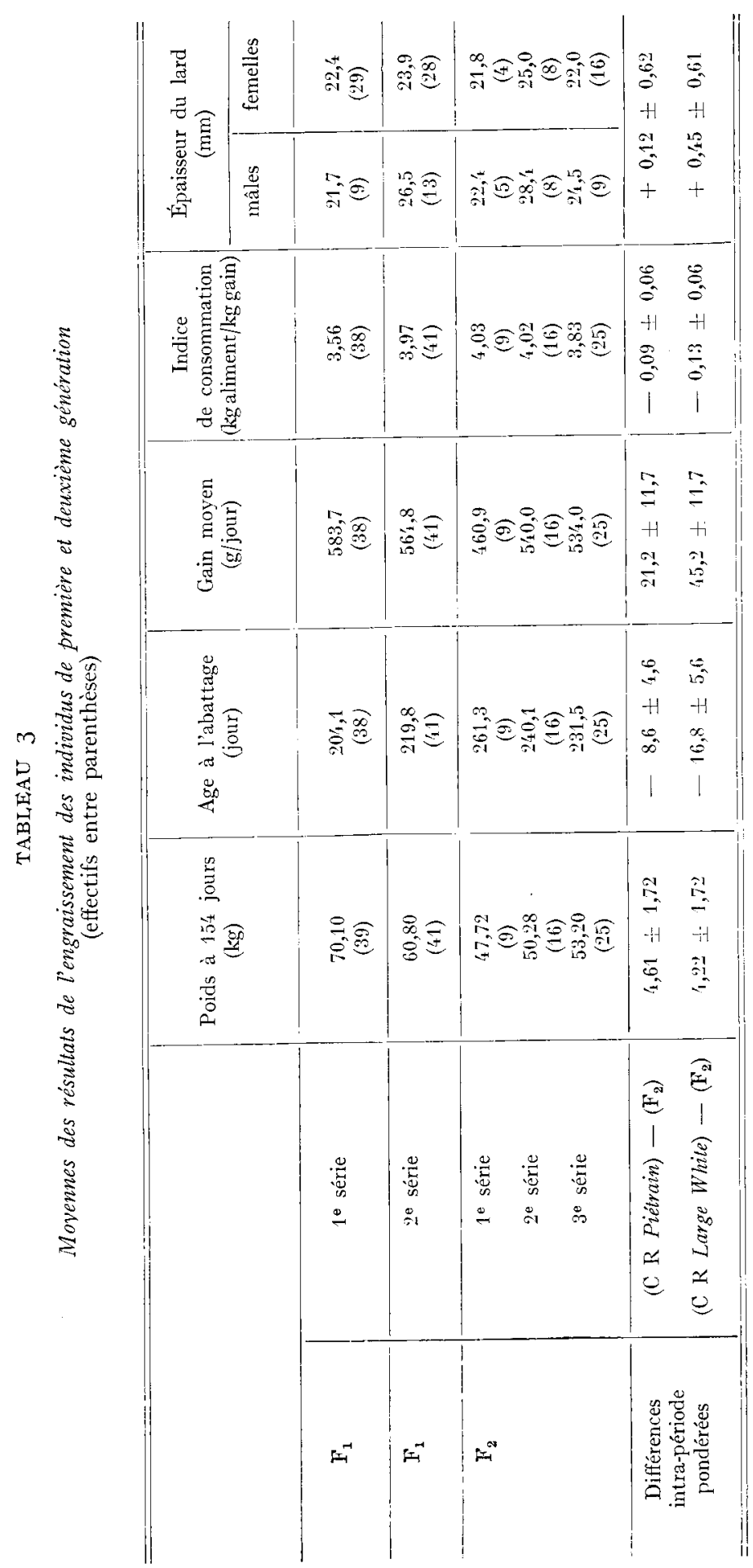




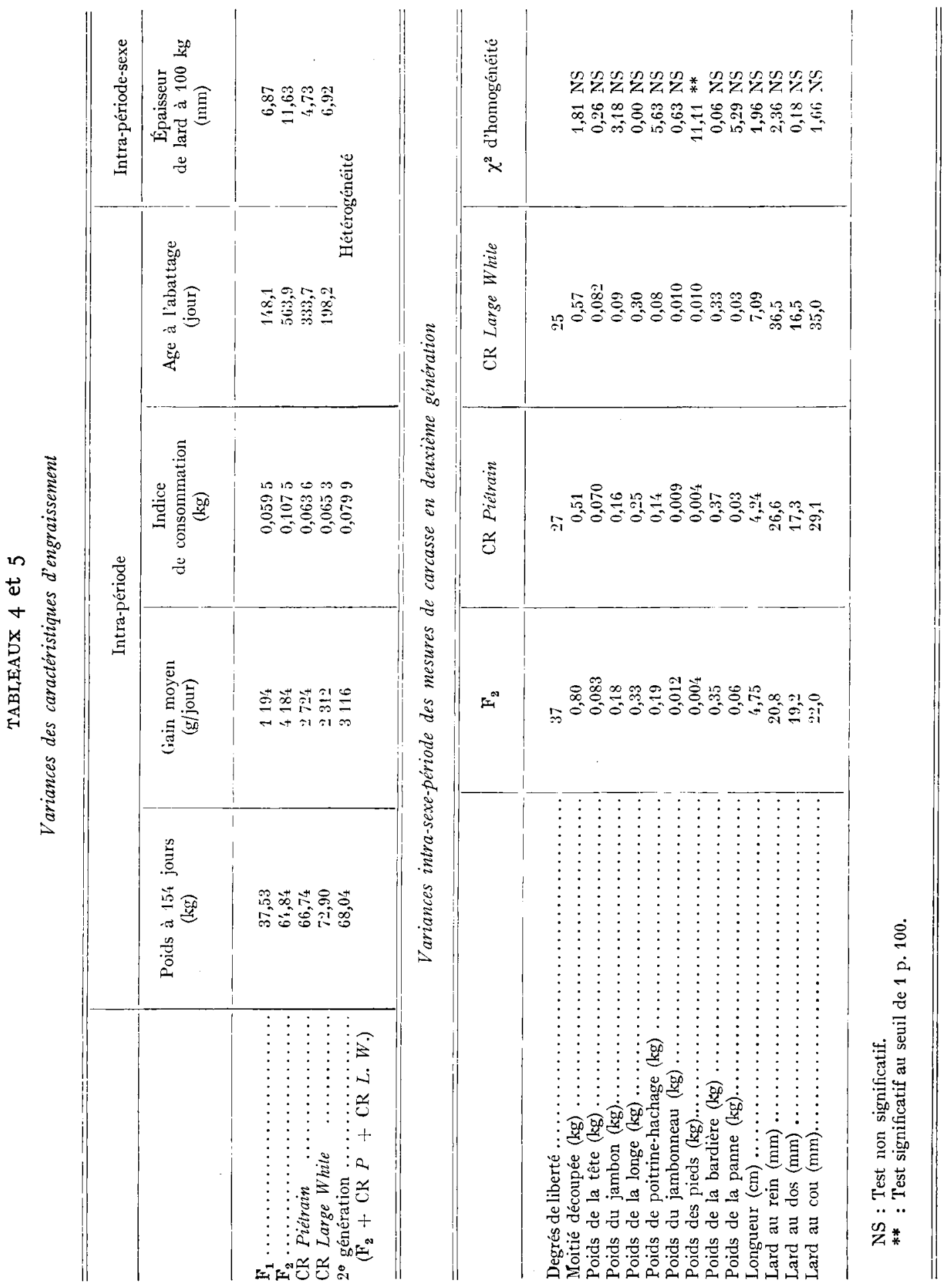


L. OLL,IVIER
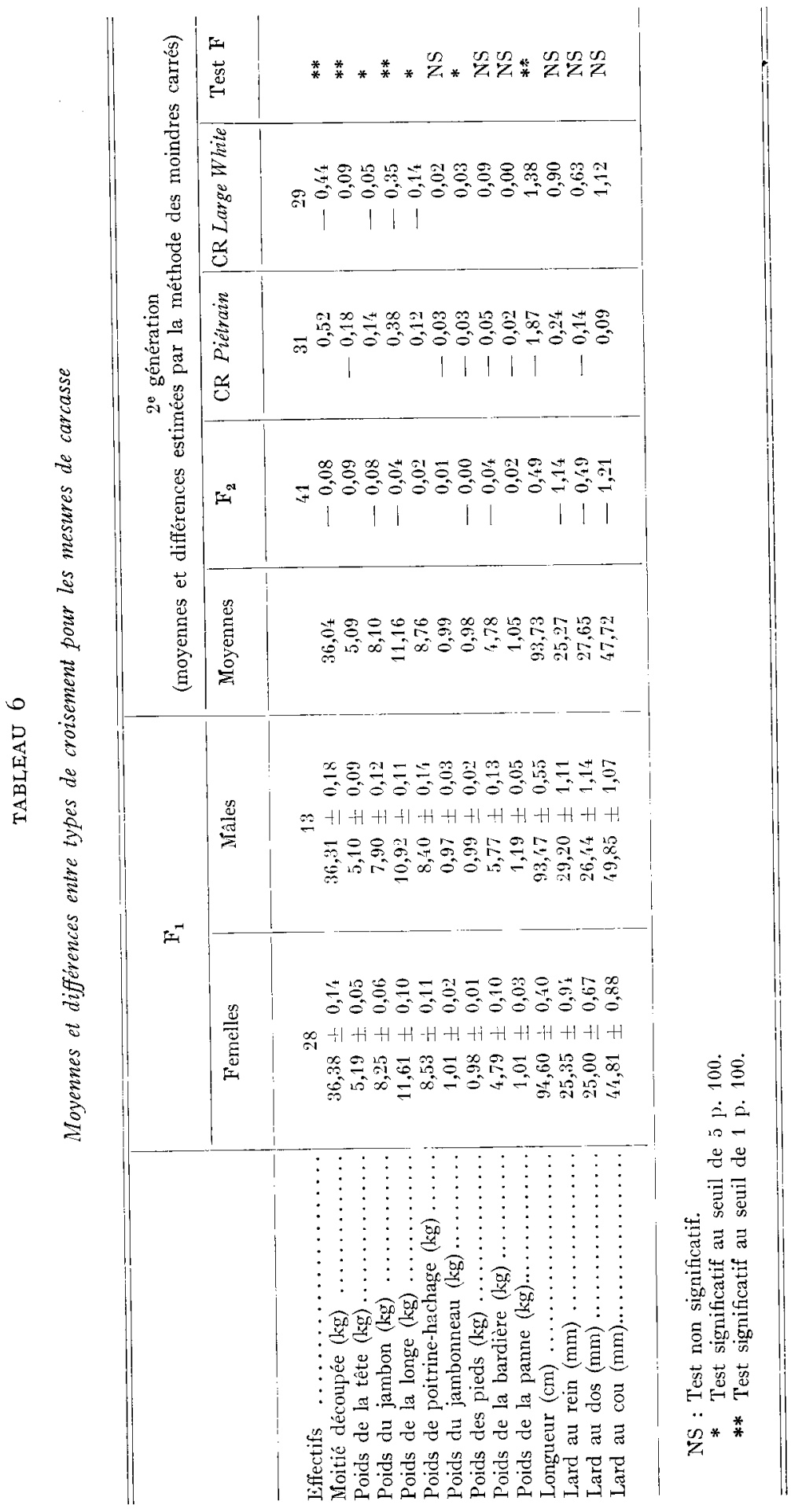
de la moitié découpée, de la tête, des pieds et des morceaux riches en viande, ainsi que sur la longueur. Par contre, toutes les mesures reliées à l'adiposité de la carcasse ne sont pas significativement influencées par le type de croisement, ce qui confirme les mesures d'épaisseur de gras effectuées sur le vivant avant l'abattage.

\section{Essai de mise en éridence d'un gène majeur ségrégeant en deuxième génération}

Les méthodes de l'analyse discriminatoire, largement employées en génétique végétale, sont d'un emploi peu fréquent en génétique animale, mise à part leur utilisation dans 1'établissement des indices de sélection. Il a paru intéressant de les appliquer aux mesures de carcasse effectuées à l'occasion de cette expérience. Le principe était d'établir d'abord une fonction linéaire permettant de discriminer au mieux les deux races parentales. Cette fonction a ensuite été appliquée aux individus de première et de deuxième génération et les distributions obtenues ont été étudiées.

a) Établissement de la fonction discriminatoire.

En l'absence de témoins contemporains des deux races parentales, un échantillon de chaque race ayant servi à l'étude de KNOERTZER (I96I) a été utilisé, soit IO9 porcs de race Large White et I04 porcs de race de Piétrain. Ces animaux ont été engraissés de $195^{8}$ à I96I à la porcherie de mise à l'épreuve des verrats sur leur descendance à Jouy-en-Josas, abattus à Ioo kg, et soumis aux mêmes mesures sur la carcasse que les animaux de cette expérience. Les I 2 variables suivantes, corrigées pour les variations du poids de la demi-carcasse par régression intra-race, ont été considérées : poids de la tête, du jambon, de la longe, de la poitrine-hachage, du jambonneau, des pieds, de la bardière et de la panne, longueur, épaisseur de lard au rein, au dos et au cou. Les effets du sexe sur chacune de ces variables ont été estimés par la méthode des moindres carrés. Pour les variables ne manifestant aucune interaction race $x$ sexe, un facteur de correction commun aux deux races a été appliqué, et, dans le cas contraire, un facteur de correction propre à chaque race a été utilisé. Le choix des variables à faire entrer dans la fonction discriminatoire a été basé sur le calcul du coefficient $D^{2}$ de Mahalanobis selon une méthode décrite par RAo (1952). Les valeurs de $\mathrm{D}^{2}$ sont d'abord calculées pour chaque variable séparément et la première variable retenue est celle ayant $1 \mathrm{l} \mathrm{I}^{2}$ le plus élevé. La deuxième variable sélectionnée est celle, parmi les variables restantes, qui donne la valeur de $\mathrm{D}^{2}$ la plus élevée en combinaison avec la première variable et ainsi de suite. I a fonction discriminatoire tenant compte des six premières variables ainsi retenues a été établie. Ces calculs ont été réalisés sur ordinateur IBM I620 $\left(^{1}\right)$, selon une méthode mise au point par WAGNER (1965). Les résultats figurent au tableau 7. La fonction choisie permet une excellente discrimination entre les deux races, puisque les deux distributions ne se recouvrent pas (fig. 2).

b) Application de la fonction discriminatoire aux produits de première et deuxième génération.

L,es distributions de chacune des six variables retenues (corrigées pour le sexe et la période comme indiqué plus haut) en deuxième génération ont d'abord été éta-

(1) Programme no 63002. Station centrale de Génétique animale, C. N. R. Z. $7^{8}$ - Jouy-en-Josas. 


\section{TABLEAU 7}

Résultals de l'analyse discriminatoire progressive sur les mesures de carcasse (races Large White et de Piétrain)

\begin{tabular}{|c|c|c|c|}
\hline $\begin{array}{l}\text { Stade } \\
\text { de } \\
\text { sélection }\end{array}$ & Variable retenue & $\begin{array}{l}\text { Valeur du coefficient } \\
\text { de la fonction discriminatoire } \\
\text { à } 6 \text { variables }\end{array}$ & $\begin{array}{l}\mathrm{D}^{2} \text { à chaque } \\
\text { stade }\end{array}$ \\
\hline 1 & Poids du jambon (dag)...... & 0,1018 & 6,28 \\
\hline 2 & Longueur $(\mathrm{mm}) \ldots \ldots \ldots \ldots$ & $-0,0792$ & 9,97 \\
\hline 3 & Poids de la longe (dag) ........... & 0,0779 & 13,61 \\
\hline it & Poids de la poitrine-hachage (dag) .. & 0,0828 & 18,85 \\
\hline 5 & Épaisseur du lard au dos (mm) ..... & $0,0 \leq 71$ & 22,13 \\
\hline 6 & Poids de la panne (dag)............. & 0,0907 & $2 / 4,43$ \\
\hline
\end{tabular}

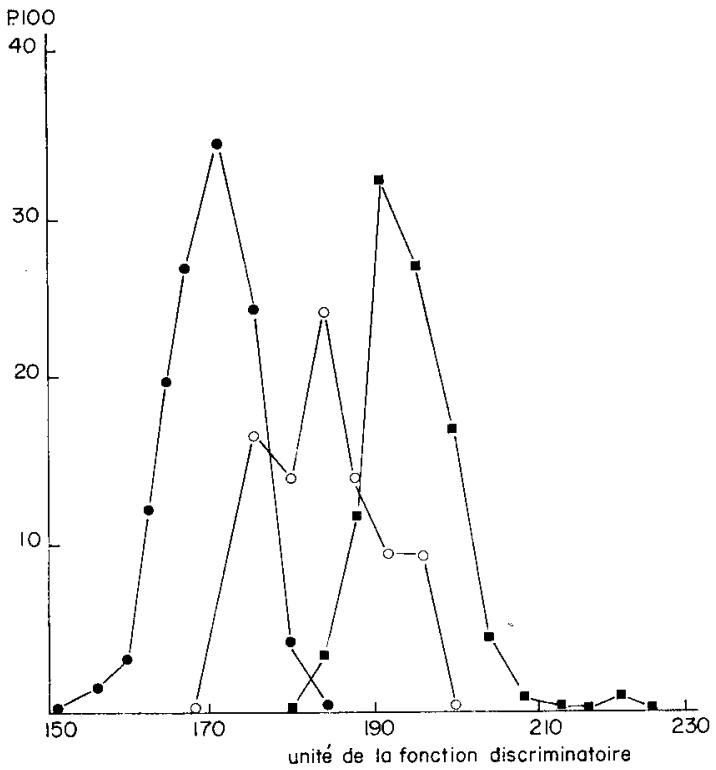

Fig. 2. - Distributions de la fonction discriminatoire pour les deux races parentales et la $F_{1}$

- Large White (Iog porcs)

- Piétrain (ro4 porcs)

$\circ \mathrm{F}_{1}(P \times L W)(4 \mathrm{I}$ porcs $)$ 
blies (fig. 3). Les distributions obtenues ne donnent guère d'indication sur le déterminisme génétique de ces caractères.
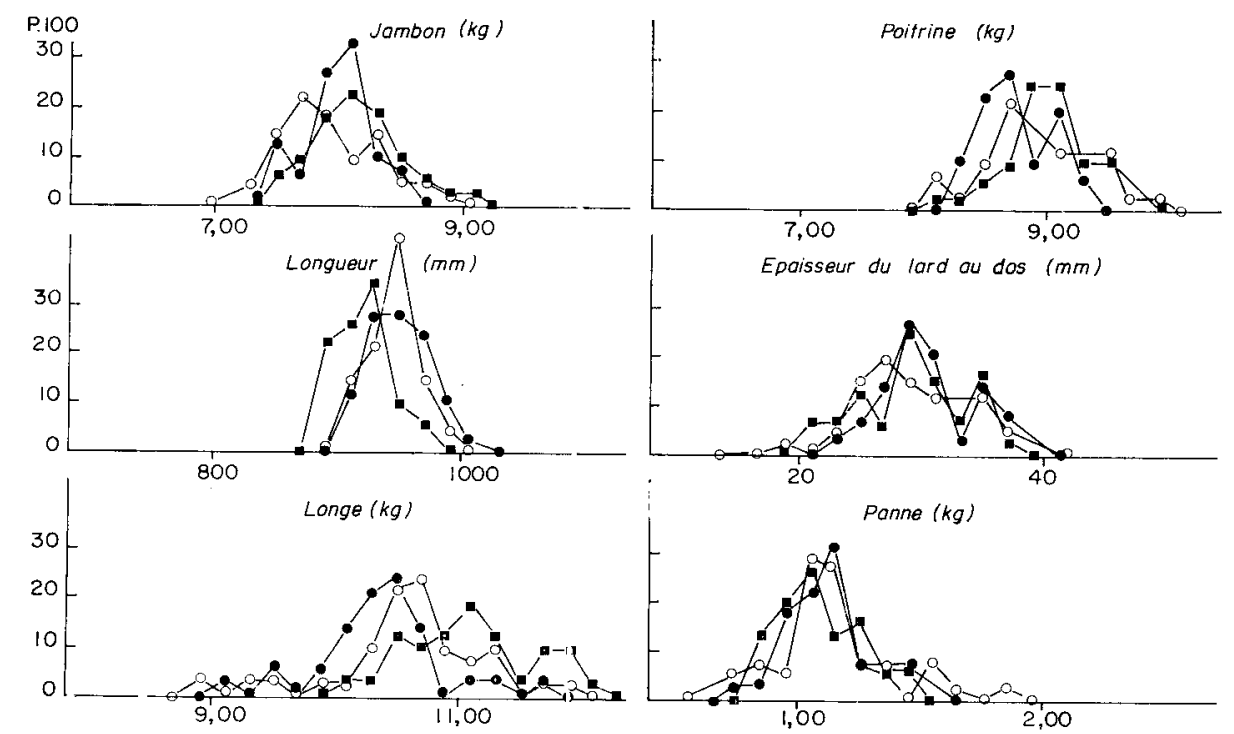

FIG. 3. - Distribulions, en deuxieme génération, des six variables de la fonction discriminatoire

- CR Large White (29 porcs)

- CR Piétrain (3I pores)

- $\mathrm{F}_{2}\left(\mathrm{~F}_{1} \times \mathrm{F}_{1}\right)$ (4I porcs)

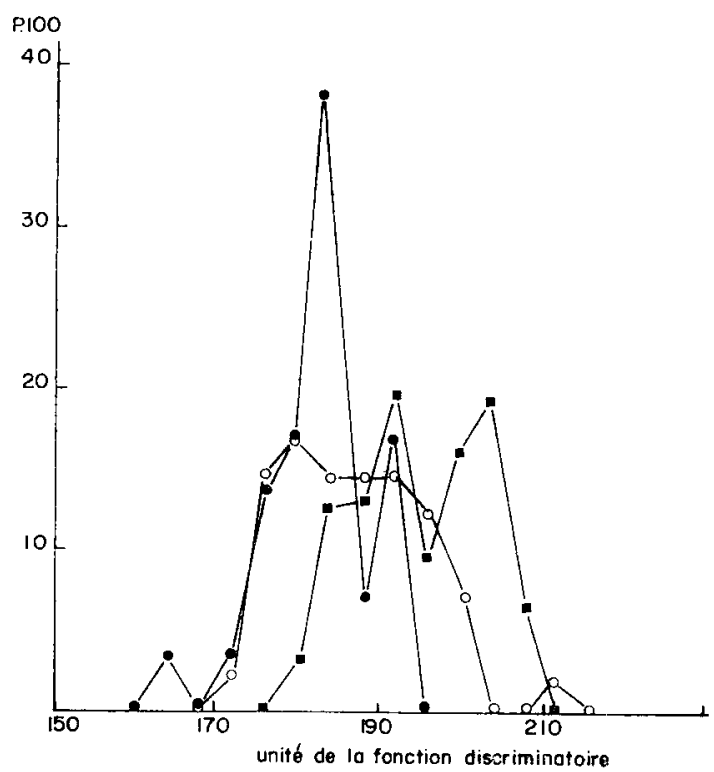

F]G. 4. - Distributions, en deuxieme génération, de la fonction discriminatoire

$$
\begin{array}{ll}
\text { - } \mathrm{CR} \text { Large While } & \text { (29 porcs) } \\
\text { - } \mathrm{CR} \text { Piétrain } & \text { (3I porcs) } \\
\text { - } \mathrm{F}_{2}\left(\mathrm{~F}_{1} \times \mathrm{F}_{1}\right) & \text { (4I porcs) }
\end{array}
$$


I a fonction discriminatoire calculée plus haut a alors été appliquée à chaque individu de première et de deuxième génération (fig. 2 et 4). Les paramètres relatifs aux distributions de cette fonction sont donnés au tableau 8. Les trois générations considérées ne sont pas contemporaines, ce qui rend les comparaisons difficiles. Nous nous bornerons donc à étudier les distributions de la $\mathrm{F}_{2}$ et des croisements en retour. L'ensemble de ces trois distributions démontre que la variation de la fonction discriminatoire a une part génétique importante. La présence de deux pics dans chacun des croisements en retour, suggérée par les échantillons observés, l'un de ces pics étant commun aux deux distributions, est compatible avec l'hypothèse d'un gène majeur responsable des différences observées entre les deux races parentales pour ce caractère. Afin de tester statistiquement cette hypothèse, les écarts à la moyenne de la fonction discriminatoire pour chaque type de croisement ont été calculés et leur distribution globale en deuxième génération a été établie. Ians 1'hy̌pothèse d'un gène majeur, comme l'a montré MírRAT (I967), une telle distribution doit être aplatie et présenter un coefficient de kurtosis négatif. Ce coefficient a été calculé : il est de $-0, I 3 \pm 0,48$. Les distributions en deuxième génération s'écartent donc de la normalité dans le sens d'un aplatissement, mais l'écart est faible et non significatif.

\section{TABIEAU $\delta$}

Parantetres des distributions de la fonction discriminatoire dans le croisement Piétrain $\times$ Large White

\begin{tabular}{|c|c|c|c|c|}
\hline & Effectif & Moyenne & Variance & Éart-type \\
\hline Race Large White... & $10^{\prime}$ & 170,17 & $\because 0,38$ & 4,51 \\
\hline Race de Piétrain.... & 109 & $19^{\prime}, 58$ & 28,24 & 5,31 \\
\hline $\mathrm{F}_{1}(L W \times P)$ & 41 & $181,2 \cdot 4$ & 59,03 & 7,68 \\
\hline $\mathrm{F}_{2}\left(\mathrm{~F}_{1} \times \mathrm{F}_{1}\right) \ldots$ & 41 & 186,85 & 69,72 & 8,35 \\
\hline $\mathrm{CR}$ Piélrain $\left(\mathrm{F}_{1} \times\right.$ Piélrain $)$ & 31 & 195,06 & $54,6=2$ & 7,39 \\
\hline CR Large White $\left(\mathrm{F}_{\mathbf{1}} \times\right.$ Large White $)$ & 29 & 183,17 & $: 3 \overline{7}, 8 \bar{z}$ & 6.15 \\
\hline
\end{tabular}

5. Relation entre le pointage de conformation au seirage et les mesures sur carcasse à l'abattage

Les distributions de la note de pointage et de la fonction discriminatoire présentent des analogies qui soulèvent la question de savoir dans quelle mesure ces deux variables sont l'expression d'un même phénomène. A fin d'y répondre, la régression. de la fonction discriminatoire en la note de pointage a été estimée sur tous les individus de la deuxième génération. Elle est de $\mathrm{r}, 40 \pm 0,33$. Il est donc possible, au sevrage, de prédire dans une certaine mesure la valeur de la fonction discriminatoire au poids d'abattage de Ioo $\mathrm{kg}$, c'est-à-dire l'intensité de la manifestation du caractère Piétrain. 


\section{DISCUSSION ET CONCLUSIONS}

L'expérience qui vient d'être décrite n'avait pas pour but de comparer les mérites zootechniques de différents types de croisement, ce qui a déjà été réalisé en ce qui concerne la race de Piétrain tant en France (KNOERTZER, I96I $b$ ) qu'à l'étranger (KIRsch et $a l$., Ig63 $a$ et $b$ ), mais d'essayer de définir objectivement une entité "Piétrain " et d'en connaître le déterminisme génétique.

La comparaison des moyennes des différents types de croisement ne permet donc pas de tirer des conclusions quant à la valeur respective des deux races utilisées. En effet, l'une d'elles est représentée par un seul verrat et l'autre par onze truies. On peut seulement faire remarquer que, pour les critères de carcasse, lorsque les différences observées sont significatives, la moyenne de la $\mathrm{F}_{\mathbf{2}}$ est intermédiaire entre celles des deux croisements en retour (sauf pour le poids de la tête et du jambon) ce qui suggère un déterminisme polygénique additif. Par contre, pour les critères de croissance, la $\mathrm{F}_{2}$ n'est jamais intermédiaire, mais a les valeurs les plus faibles. Il est probable que la consanguinité élevée de la $\mathrm{F}_{2}(\mathrm{~F}=0,25)$ par rapport au croisement en retour au Piétrain $(\mathrm{F}=0, \mathrm{I25})$ est responsable de cette situation. Les effets dépressifs de la consanguinité sur la croissance du porc sont en effet bien connus.

Si nous considérons individuellement chacun des caractères mesurés dans cette expérience, les distributions observées en deuxième génération ne permettent pas de mettre en évidence l'existence d'un gène majeur. Par contre, le pointage de la conformation au sevrage fait apparaître trois catégories d'animaux en deuxième génération, qu'on trouve représentées dans le croisement $F_{2}$, mais dont deux seulement figurent dans chacun des croisements en retour. Une telle répartition suppose un déterminisme génétique simple. On retrouve les tendances qui se dégageaient dans l'étude préliminaire (OLLIVIER et LAUVERGNE, I967).

D'autre part, les distributions d'une combinaison linéaire de six mesures de carcasse, obtenue par analyse discriminatoire, présentent des irrégularités telles qu'il devrait s'en produire si un gène majeur ségrégeait en deuxième génération. Une méthode analogue a permis à WEBER (I959) de démontrer la ségrégation d'un couple de gènes, à partir d'une fonction discriminatoire incluant deux mesures du cotylédon chez la Tomate. En ce qui concerne notre expérience sur le Porc, les effectifs observés en deuxième génération sont malheureusement trop réduits pour pouvoir aboutir à une conclusion aussi nette. Le nombre de gènes intervenant dans le déterminisme de la fonction discriminatoire, estimé par l'indice de ségrégation de WRIGHT (I952), serait de $I, 6$. Ce chiffre n'est toutefois donné qu'à titre indicatif, à cause des nombreuses hypothèses restrictives nécessaires à la validité de la formule appliquée.

La convergence des résultats de l'examen visuel de la conformation et des mesures objectives de carcasse permet de penser qu'une sélection sur la conformation au sevrage a pu être à l'origine đu développement rapide de cette race très différente dans ses caractéristiques de composition corporelle de toutes les autres races porcines européennes.

L'amélioration des productions animales repose actuellement sur la sélection en faveur de gènes nombreux, non identifiés, et dont les effets individuels sur les 
caractères à sélectionner sont supposés faibles. Une tendance récente consiste à étudier les effets de gènes facilement identifiables dont l'utilisation en sélection pourrait apporter un surcroît de gain génétique appréciable, comme l'a démontré Smitu (Ig67).

Notre étude suggère l'existence d'une troisième catégorie de gènes utiles à l'élevage, gènes majeurs à très forte action potentielle mais dont la mise en évidence est difficile et qui, par ailleurs, s'accompagnent souvent d'effets pléiotropiques défavorables. On peut, comme nous l'avons fait, rechercher une combinaison linéaire de mesures se rapprochant le plus possible de l'effet du gène postulé. Mais dans le cas du porc de Piétrain, comme dans celui analogue du culard bovin, il reste encore à mettre au point un test vraiment spécifique de 1'entité observée, avant de poursuivre les études génétiques.

Reçu pour publication en septembre 1968.

\section{REMERCIEMEN'TS}

L'auteur tient à exprimer sa plus vive reconnaissance à M. J.- J. Latjvergne, qui a eu l'idée de cette expérience et a collaboré à la mise sur pied du dispositif expérimental.

\section{SUMMARY}

A STUDY OF THE GENETIC DETERMINATION OF MUSCULAR IIYPERTROPHY

IN THE " PIÉTRAIN" PIG

From a Piétrain boar and cleven Large White sows, a first generation cross $\left(\mathrm{F}_{\mathbf{1}}\right)$ was obtained, then a $\mathrm{F}_{2}\left(\mathrm{~F}_{\mathrm{I}} \times_{1}\right)$ and the back-crosses $(\mathrm{BC})$ to the two parental breeds, in order to test the hypothesis of a monogenic determination of muscular hypertrophy in the Pietrain pig.

The $F_{1}, F_{2}$ and $\mathrm{BC}$ animals were visually given a score for muscular development at weaning, $i . e$. when they were two months old (406 pigs). Out of these 220 pigs were submitted to various measures during fattening (between 25 an Ioo kg liveweight) and I 42 pigs were slaughtered in order to take measurements on the carcass.

The distribution of the visual score in the second generation $\left(\mathrm{F}_{2}\right.$ and $\left.\mathrm{BC}\right)$ suggests the existence of three categories of individuals. On the other hand, no objective measure considered alone shows the existence of distinct categories. However, a linear combination of six measurements on the carcass - a discriminant function established on the two parental breeds - presents some irregularities in its distribution analogous to those observed for visual score. Moreover, a significant regression of the discriminant function as defined above exists on the score at weaning. Altogether the results suggest that the number of genes responsible for muscular hypertrophy in the Pietrain breed is limited and that those genes express themselves from the age of two months.

\section{RÉFÉ,RENCES BIBIIOGRAPHIQUES}

Camerlynck R., I962. Le porc Piétrain à l'étranger. Revue agric., Brex., 15, 237-275.

Camerlynck R., Brankaer R., 1958. Quelques considérations sur la race porcine Piétrain en Belgique. Revue agric., Brux., 11, 379-399.

Castle W. E., r921. An improved method of estimating the number of genetic factors concerned in cases of blending inheritance. Science, 54, 93 . 
Dehaye L., 1962. Dix années de sélection et d'observation de la race porcine Piétrain. Revue agric., Brux., 15, 35-52.

HAZEL L. N., KLINE E. A., 1952. Mechanical measurement of fatness and carcass value on live hogs. J. Anim. Sci., 11, 3I3-318.

Kirsch W., Fender M., Rabold K., Fewson D., Schoen P., I963a. Vergleichende Zucht-, Mast-und Ausschlachtungsversuche mit veredelten Landschweinen, Piétrain-Schweinen und $\mathrm{F}_{1}$ Kreuzungstieren. Züchtungskunde, 35, 254-264.

Kirsch W., Fewson D., Fender M., Werkmeister F., I963 $b$. Kombinationszüchtung und Zuchtlinien Kreuzung als Möglichkeit zur Ausnutzung der erwünschten Eigenschaften der Piétrain-Schweine. Zïchingskunde, 35, 305-315.

Knoertzer. E., i96r a. Quelques données biométriques sur le porc de Piétrain. Bull. tech. Inf. Ingrs Servs agric., no 164, nov. I 96 I.

Knoertzer li., ig6r $b$. Le croisement porcin Piétrain $\times$ Large While. Bull. tech. Inf. Ingrs Servs agric., 165, déc. 1961 .

Lauvergne J.-J., Vissac B., Perramon A., ig63. Étude du caractère culard I. Mise au point bibliographique. Annls Zootech., 12, I33-г $5^{6 .}$

MÉrat P., Ig66. Contribution à l'étude de la "valeur sélective " associée à quelques gènes chez la Poule domestique. I. Annls Biol. anim. Bioch. Biophys., 1, 79-I04.

MÉrat P., I967. L'étude des distributions de fréquence en vue de déceler la présence de gènes à effets importants. Annls Biol. anim. Bioch. Biophys. (sous presse).

Ollivier L., I964: Note sur le sondage du gras dorsal chez le porc vivant à l'aide d'une réglette métalliqueC. R. Acad. Agric. Fr., 50, 656-66r.

Ollivier L., Lauvergne J. J., I967. Étude du déterminisme héréditaire de l'hypertrophie musculaire du porc de Piétrain : premiers résultats. Annls Méd. vét., 111, I04-109.

RAO R., I952. Advanced statistical methods in biometric research. John Wiley. New York.

Smith C., I967. Improvement of metric traits through specific genetic loci. Anim. Prod., 9, 349-358.

SEREBRovSKy A. S., I928. An analysis of the inheritance of quantitative transgressive characters. $Z$. indukl. Abstamm. u. VererbLehre, 48, 229-243.

THODAY J. M., 196r. Location of polygenes. Nature, 151, 368-370.

Wagner R., I965. Sélection progressive de variables utilisant la statistique $\mathrm{D}^{2}$ de Mahalanobis. Application à la détermination de la meilleure fonction discriminatoire séparant deux populations de semis de vigne. Annls Amél., Pl., 15, 159-181.

WEBER E., I959. The genetical analysis of characters with continuous variability on a mendelian basis. I. Monohybrid segregation. Genetics, 44, I I 3 I-I I 39 .

Willeys A. L. R., I960. Origine et évolution du porc belge de Piétrain. Colloque sur la race parcine belge Piétrain organisée par la F. E. Z.

WRIEDT C., I930. The inheritance of butter fat percentage in crosses of fersey with Red Danes. J. Genet., 22, $45-53$.

WRIGHT S., 1952. The genetics of quantitative variability. In Quantitative Inheritance 5-41. Her Majesty's Stationery Office. Londres. 